\title{
Identification of the Foundation Stiffness of Large Structure as an Inverse Problem*
}

\author{
Toshimichi FUKUOKA**, Masataka NOMURA** and Yuyong ZHAO** \\ ${ }^{* *}$ Faculty of Maritime Sciences, Kobe University \\ 5-1-1 Fukaeminami, Higashinada-ku, Kobe, Hyogo, 658-0022, Japan \\ E-mail: fukuoka@maritime.kobe-u.ac.jp
}

\begin{abstract}
When analyzing the strength of large structures, the stiffness of the foundation, on which the structure is placed, might significantly affect the numerical results. In this paper, a finite element approach to estimate the foundation stiffness as an inverse problem is proposed, where the target large structure is placed on the elastic foundation that are modeled as groups of one-dimensional spring elements in parallel. The magnitude of the spring constant that represents the foundation stiffness can exactly be calculated algebraically by use of the same number of measured surface deflections. In the numerical analyses, unknowns are the diagonal components in the global stiffness matrix that include the aforementioned spring constants. The validity and the accuracy of the proposed numerical method are verified by comparing the numerical results to the exact solutions for bending problems of a cantilever supported by a single spring element.
\end{abstract}

Key words : Foundation Stiffness, Inverse Problem, FEM, Spring-Supported Boundary, Cantilever

\section{Introduction}

When large machines or structures are placed on the foundation whose deflection cannot be ignored, the foundation stiffness significantly affects the numerical accuracy in the case of analyzing their mechanical behavior caused by the external force or the deflection due to its own weight. It is a common practice to represent the foundation stiffness as a spring-supported boundary when analyzing the strength and the stiffness of large structures or machines by FEM. However, it is not necessarily easy to estimate the magnitude of spring constants with sufficient accuracy. Meanwhile, a laser displacement sensor has recently come to be used widely, which can indirectly measure the surface deflection of various-sized structures with high accuracy. If the foundation stiffness of the large structure, which has already been placed on the base, can be estimated as an inverse problem by measuring the surface deflections, it is possible to precisely evaluate the stress distribution due to its own weight and the mechanical behavior caused by the external force in service condition.

In this study, supposing a FE models of a large structure of which surface deflections are known at several nodes, a finite element approach to estimate the foundation stiffness as an inverse problem is proposed by modeling the elastic foundation as groups of spring elements $^{(1),(2)}$. In order to evaluate the foundation stiffness with high accuracy, it is necessary to obtain the stiffness both in the normal and tangential directions. Although the present method is effective to calculate the stiffness in both directions simultaneously, only the normal stiffness is treated here for simplicity, since it has a dominant effect on the mechanical behavior of the target structure. The foundation stiffness is quantitatively expressed in terms of groups of one-dimensional spring elements. Since the same number of spring elements as the known nodal surface deflections can be incorporated into FE model, the numerical accuracy for the foundation stiffness increases as the measuring points of surface deflections are increased. 


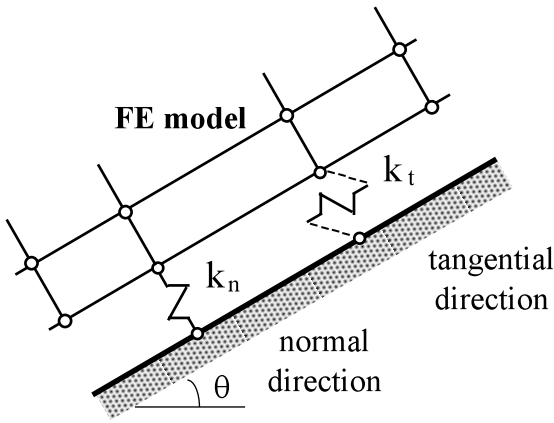

Fig. 1 Spring-supported boundaries

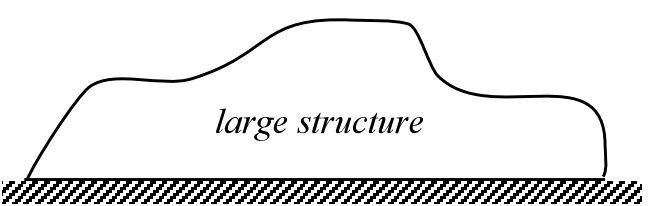

(a) Rigid foundation

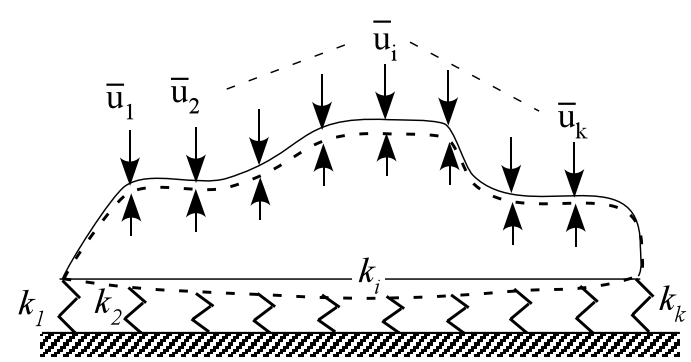

(b) Elastic foundation

Fig. 2 Large structures placed on rigid and elastic foundations

In FE models with spring-supported boundaries, each spring constant of one-dimensional spring element that represents the foundation stiffness is added to the corresponding diagonal component in the global stiffness matrix. Therefore, the proposed method in this study reduces to solve a set of $n$ linear algebraic equations with $k$ known nodal displacements, $k$ unknown diagonal components in the global matrix and $(n-k)$ unknown nodal displacements.

\section{Large Structures placed on Elastic Foundation}

\subsection{Structure with spring-supported boundaries}

When analyzing the strength and stiffness of large structures by FEM, the deformation of the foundation due to its own weight sometimes becomes a serious problem. For example, the effect of the foundation deformation cannot be ignored for such cases as a large-sized diesel engine placed in the ship engine room and a petroleum reserving tank. The lower surfaces of those structures are often modeled as spring-supported boundaries. Figure 1 illustrates the nodal points supported by spring elements on the surface, with inclination angle of $\theta$ measured from the horizontal surface. $k_{n}$ and $k_{t}$ in the figure represent the spring constants in the normal and tangential directions. In the case of two dimensional problems, the spring-supported boundary can be incorporated into the finite element formulation by adding the following terms to the corresponding nodal components in the global matrix $[K]^{(3)}$.

$$
k_{n}\left[\begin{array}{cc}
\sin ^{2} \theta & -\sin \theta \cos \theta \\
-\sin \theta \cos \theta & \cos ^{2} \theta
\end{array}\right]+k_{t}\left[\begin{array}{cc}
\cos ^{2} \theta & \sin \theta \cos \theta \\
\sin \theta \cos \theta & \sin ^{2} \theta
\end{array}\right]
$$

It is obvious from the above equation that in the case of $\theta=0, k_{n}$ and $k_{t}$ are to be simply added to the corresponding diagonal components because $\sin \theta=0$ and $\cos \theta=1$ for $\theta=0$. Figure 2(a) illustrates a large structure placed on the rigid foundation. In this case, the deformation of the structure due to its own weight can generally be neglected. On the contrary, when the same structure is placed on the elastic foundation, shown by the broken line in Fig. 2(b), the structure deformation caused by the foundation deflection often cannot be ignored. That is because the foundation deforms due to the weight of the structure. There exist two kinds of the foundation stiffness, i.e., in the normal and tangential directions, as shown in Fig. 1. In Fig. 2(b), only the normal stiffness is depicted, which might have a dominant effect when analyzing the strength of large structures. $k_{i}(i=1,2, \ldots, k)$ is the one-dimensional spring constant that represents the normal stiffness of the foundation, and it corresponds to $k_{n}$ in Fig. 1. $\bar{u}_{i}(i=1,2, \ldots, k)$ is the normal deflection on the structure surface. Each component of $\bar{u}_{i}$ is a known value indirectly measured by laser displacement sensors. Assuming that $n$ is 
the total degrees of freedom of the objective FE model, the global stiffness equation reduces to a set of $n$ algebraic equations.

$$
[K]\{u\}=\{f\}
$$

In the above equation, $k$ components of the displacement vector $\{u\}$ are known and $k$ diagonal components of the global stiffness matrix $[K]$ and $(n-k)$ components of $\{u\}$ are unknown. In the case of two-dimensional problem, the spring constant $k_{i}$ represents the stiffness in the y direction. It is added to the diagonal component $k_{2 i} 2 i$ in $[K]$, thus $\left(k_{2 i} 2 i+k_{i}\right)$ becomes the unknown diagonal component to be calculated. When evaluating the tangential stiffness, $k_{t}$ is added to the diagonal component $k_{2 i-12 i-1}$ in $[K]$, and the magnitude of each $k_{t}$ is calculated following the same manner as in the case of $k_{n}$. That is, the number of known displacements needs to be increased according the number of tangential spring elements.

\subsection{Relationship between known displacements and deformation of large structure}

The surface deflections of large structures can be measured at a number of locations indirectly and simultaneously by means of laser displacement sensors. The amounts of measured deflections are transferred into known displacements $\bar{u}_{i}$ appeared in Eq. (2) in the following manner.

Step1: The surface configuration before deformation due to the foundation deflection is set to be the reference shape.

Step2: The target large structure is placed on the elastic foundation. Its surface deflections are measured by moving the laser displacement sensor parallel to the foundation. The deflections from the reference shape are set to be known displacements $\bar{u}_{i}$ in Eq. (2).

A simple method to define the reference shape is to employ the design shape of the objective structure, which is measured with reference to the foundation surface. On the other hand, a certain amount of machining and assembly errors inevitably occurs. Therefore, the reference shape is expectantly determined by measuring the configuration of the structure being placed on the rigid foundation. In Fig. 2(b), the solid line and broken one show the structure profiles before and after deformation, respectively. The difference between the two profiles is to be a known displacement $\bar{u}_{i}$. It is also evident from the above explanation that the proposed method deals with the structures whose rigid deformation is restrained.

\section{Method for Calculating Unknown Diagonal Components in Simultaneous Al- gebraic Equation}

The proposed method in this study aims at solving a set of $n$ linear algebraic equations with $k$ unknown diagonal components in the matrix of the coefficients and $(n-k)$ unknowns and $k$ known components in the vector of known quantities. The unknown diagonal component is the sum of $k_{2 i} 2 i$ and $k_{i}$, as explained in the foregoing chapter, where the former represents the original stiffness and the later the foundation stiffness. In the first place, therefore, the sum of $\left(k_{2 i} 2 i+k_{i}\right)$ is calculated as an unknown diagonal component. The spring constant $k_{i}$, which represents the foundation stiffness, is simply obtained by subtracting $k_{2 i} 2 i$ from $\left(k_{2 i} 2 i+k_{i}\right)$. In the following, the basic idea of the proposed method is explained using a set of 4 algebraic equations. Let $\hat{a}_{22}$ be an unknown diagonal component in $[A]$ and $\bar{x}_{3}$ a known component in $\{u\}$.

$$
\left[\begin{array}{llll}
a_{11} & a_{12} & a_{13} & a_{14} \\
a_{21} & \hat{a}_{22} & a_{23} & a_{24} \\
a_{31} & a_{32} & a_{33} & a_{34} \\
a_{41} & a_{42} & a_{43} & a_{44}
\end{array}\right]\left\{\begin{array}{l}
x_{1} \\
x_{2} \\
\bar{x}_{3} \\
x_{4}
\end{array}\right\}=\left\{\begin{array}{l}
b_{1} \\
b_{2} \\
b_{3} \\
b_{4}
\end{array}\right\}
$$

As a first step, Eq. (3) is transformed to calculate unknown vector components, $x_{1}, x_{2}, x_{4}$ by 


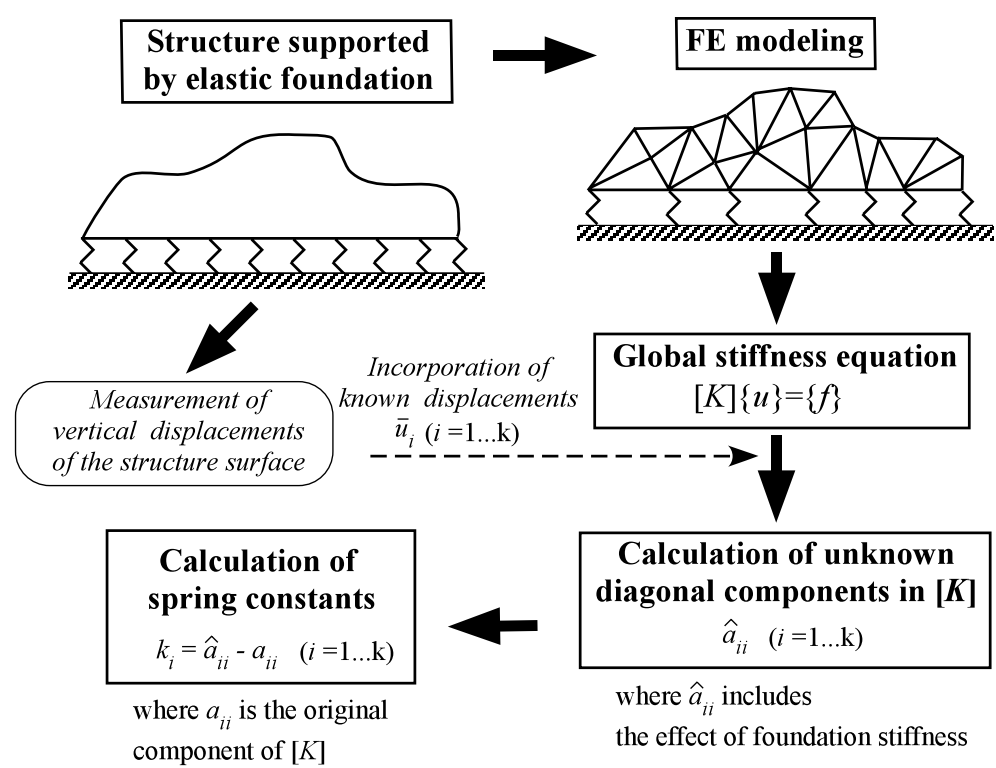

Fig. 3 Calculation procedure of spring constants representing the foundation stiffness

removing the row including $\hat{a}_{22}$ and the column including $\bar{x}_{3}$ in the coefficient matrix $[A]$.

$$
\left[\begin{array}{lll}
a_{11} & a_{12} & a_{14} \\
a_{31} & a_{32} & a_{34} \\
a_{41} & a_{42} & a_{44}
\end{array}\right]\left\{\begin{array}{l}
x_{1} \\
x_{2} \\
x_{4}
\end{array}\right\}=\left\{\begin{array}{l}
b_{1}-a_{13} \bar{x}_{3} \\
b_{3}-a_{33} \bar{x}_{3} \\
b_{4}-a_{43} \bar{x}_{3}
\end{array}\right\}
$$

Equation (4) can be easily solved following the usual manner and $x_{1}, x_{2}, x_{4}$ are obtained. Substituting the three vector components and $\bar{x}_{3}$ into Eq. (3) and transforming the row including $\hat{a}_{22}$, the unknown diagonal component $\hat{a}_{22}$ is calculated as follows.

$$
\hat{a}_{22} x_{2}=b_{2}-\left(a_{21} x_{1}+a_{23} \bar{x}_{3}+a_{24} x_{4}\right)
$$

When two or more unknown diagonal components exist, they can be calculated just by repeating the same process for each unknown.

Next, the foregoing procedure is generalized so that it can be applied to a set of $n$ algebraic equations. Considered is the case with $k$ unknown diagonal components and $k$ known vector ones.

$$
[A]\{x\}=\{b\}
$$

$[A]$ is the matrix of coefficient, $\{x\}$ vector of unknowns, and $\{b\}$ known quantities. Letting $a_{i j}$, $x_{i}, b_{i}$ be the components of $[A],\{x\}$ and $\{b\}$, Eq. (6) is rewritten as follows.

$$
\left[\begin{array}{cccc}
a_{11} & a_{12} & \cdots & a_{1 n} \\
a_{21} & a_{22} & \cdots & a_{2 n} \\
\vdots & \vdots & \ddots & \vdots \\
a_{n 1} & a_{n 2} & \cdots & a_{n n}
\end{array}\right]\left\{\begin{array}{c}
x_{1} \\
x_{2} \\
\vdots \\
x_{n}
\end{array}\right\}=\left\{\begin{array}{c}
b_{1} \\
b_{2} \\
\vdots \\
b_{n}
\end{array}\right\}
$$

where $\bar{x}_{j_{l}}(l=1,2, \ldots, k)$ and $x_{j_{m}^{\prime}}(m=1,2, \ldots, n-k)$ represent the known and unknown components of $x_{i}$. Changing Eq. (7) and collecting the terms relating to $x_{j_{m}^{\prime}}$ in the left side and 


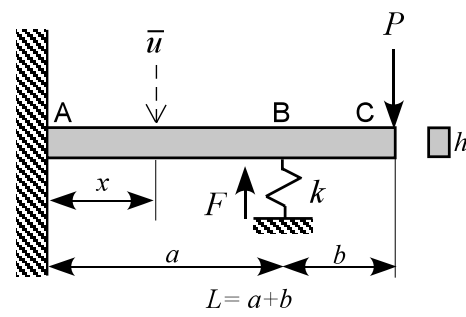

(a) Model-1

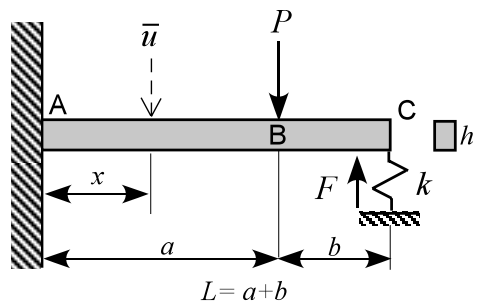

(b) Model-2

Fig. 4 Cantilevers with spring-supported boundaries

those relating to $\bar{x}_{j_{l}}$ in the right side, the generalized form of Eq. (4) is derived as follows.

$$
\left[\begin{array}{cccc}
a_{j_{i}^{\prime} j_{1}^{\prime}} & a_{j_{1}^{\prime} j_{2}^{\prime}} & \cdots & a_{j_{1}^{\prime} j_{n-k}^{\prime}} \\
a_{j_{2}^{\prime} j_{1}^{\prime}} & a_{j_{2}^{\prime} j_{2}^{\prime}} & \cdots & a_{j_{2}^{\prime} j_{n-k}^{\prime}}^{\prime} \\
\vdots & \vdots & \ddots & \vdots \\
a_{j_{n-k}^{\prime} j_{1}^{\prime}} & a_{j_{n-k}^{\prime} j_{2}^{\prime}} & \cdots & a_{j_{n-k}^{\prime} j_{n-k}^{\prime}}^{\prime}
\end{array}\right]\left\{\begin{array}{c}
x_{j_{1}^{\prime}} \\
x_{j_{2}^{\prime}} \\
\vdots \\
x_{j_{n-k}^{\prime}}
\end{array}\right\}=\left\{\begin{array}{c}
b_{j_{1}^{\prime}}-\sum_{l=1}^{k} a_{j_{1}^{\prime} j_{l}} \bar{x}_{j_{l}} \\
b_{j_{2}^{\prime}}-\sum_{l=1}^{k} a_{j_{2}^{\prime} j_{l} \bar{x}_{j_{l}}} \\
\vdots \\
b_{j_{n-k}^{\prime}}-\sum_{l=1}^{k} a_{j_{n-k}^{\prime} j_{l} \bar{x}_{j_{l}}}
\end{array}\right\}
$$

Now, all the components of the coefficient matrix $[A]$ and the vector in the right side $\{b\}$ are known. Then, the unknown components $x_{j_{m}^{\prime}}$ are calculated by solving Eq. (8). At this stage, all the components of $x_{i}$ are obtained. Subsequently, the unknown diagonal components $a_{i_{l} i_{l}}(l=1,2, \ldots, k)$ are calculated from the following equation.

$$
a_{i_{l} i_{l}} x_{i_{l}}=b_{i_{l}}-\sum_{m \neq i_{l}} a_{i_{l} m} x_{m}
$$

Equation (9) is the generalized form of Eq. (5). It is obvious from the foregoing procedure that the calculated diagonal components $a_{i i_{l} i_{l}}$ are algebraically exact solutions for the given simultaneous equations. The errors involved in the solutions are numerical errors due to the mesh size of FE model and the numerical integration scheme. Figure 3 schematically illustrates the proposed calculation procedure. $\bar{u}_{i}$ is the known displacement. $a_{i i}$ is the original diagonal component and $\hat{a}_{i i}$ denotes the unknown one that includes the spring constant $k_{i}$.

\section{Validity of the Proposed Calculation Procedure and Numerical Examples}

\subsection{Cantilever supported by a single spring element}

To demonstrate the effectiveness of the proposed method in this study, some problems of cantilever with one spring-supported boundary are solved. Figure 4 shows two cases of the problems. In the first problem, the spring is placed between the fixed end and the loading point, and in the second one, the spring is placed beyond the loading point. Although examples should be preferably selected to be consistent with the case of large structures, i.e., the deflection occurs due to its own weight, the deformation in this case is simply given by a concentrated load.

The problems shown in Fig. 4 can be easily solved when the spring constant $k$ and the magnitude of concentrated load $P$ are given. In the case of Model-1, e.g., the difference between the deflections at Point $\mathrm{B}$ due to load $P$ and spring reaction force $F,\left.y_{\mathrm{B}}\right|_{P}-\left.y_{\mathrm{B}}\right|_{F}$, is equal to the spring deflection $F / k$.

$$
\left.y_{\mathrm{B}}\right|_{P}-\left.y_{\mathrm{B}}\right|_{F}=\frac{F}{k}
$$

Applying the theoretical equations of cantilever deflection $\left.y_{\mathrm{B}}\right|_{P}$ to $\left.y_{\mathrm{B}}\right|_{F}$ and in Eq. (10), the expressions for calculating the spring reaction force $F$ and the deflection at each point $y$ along the length can be derived. The resulting equations are given in the following, in which 


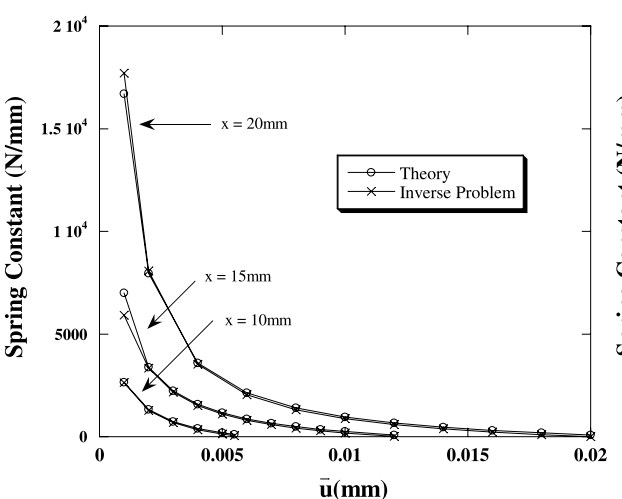

(a) Model-1

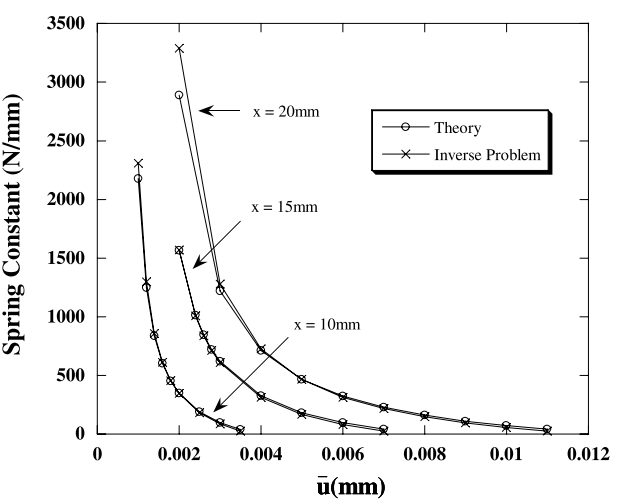

(b) Model-2

Fig. 5 Relationship between spring constants and prescribed displacements
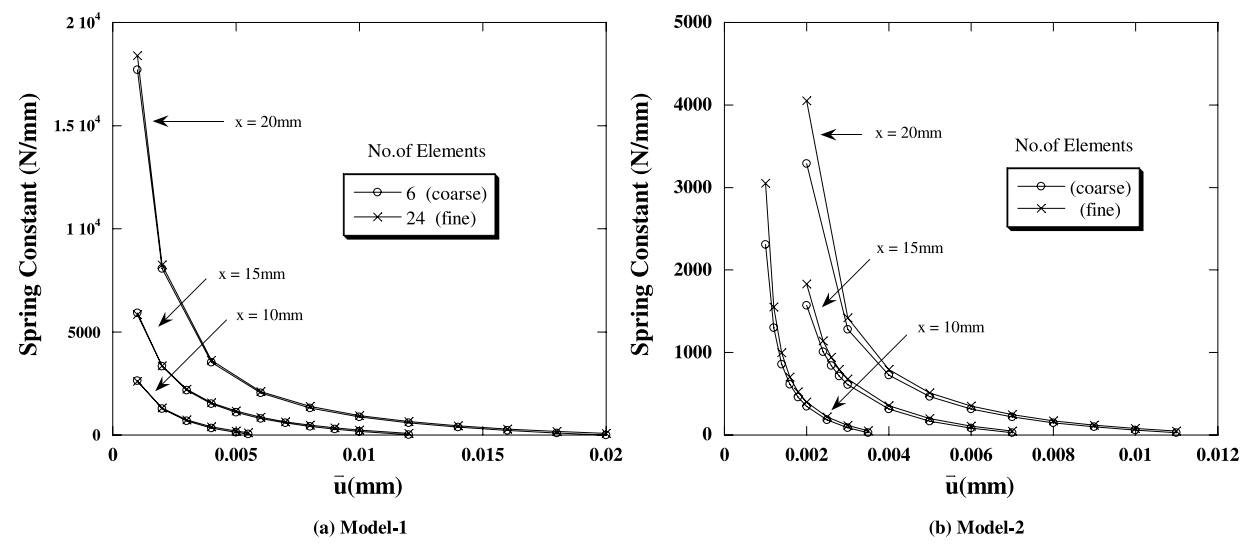

Fig. 6 Effect of mesh size on the accuracy of numerical results

$E$ and $I$ are Young's modulus and second moment of area, and $y_{\mathrm{A}-\mathrm{B}}$ and $y_{\mathrm{B}-\mathrm{C}}$ represent the deflections between Point-A and Point-B and between B and C, respectively.

$<$ Model-1>

$$
\begin{aligned}
& F=\frac{P a^{2}(2 a+3 b)}{2 a^{3}+\frac{6 E I}{k}} \\
& y_{\mathrm{A}-\mathrm{B}}=\frac{x^{2}}{6 E I}\{P(3 L-x)-F(3 a-x)\} \\
& y_{\mathrm{C}-\mathrm{D}}=\frac{1}{6 E I}\left\{P(x+a)^{2}(3 L-a-x)-F a^{2}(2 a+3 x)\right\}
\end{aligned}
$$

$<$ Model-2>

$$
\begin{aligned}
& F=\frac{P a^{2}(2 a+3 b)}{a^{2}(2 a+3 b)+\frac{6 E I}{k}} \\
& y_{\mathrm{A}-\mathrm{B}}=\frac{x^{2}}{6 E I}\{P(3 a-x)-F(3 L-x)\} \\
& y_{\mathrm{C}-\mathrm{D}}=\frac{1}{6 E I}\left\{P a^{2}(2 a+3 x)-F(x+a)^{2}(3 L-a-x)\right\}
\end{aligned}
$$

Using the above equations, the spring constant $k$ at the supported point can be calculated for a known displacement $\bar{u}$. In the case of Model-1, the reaction force $F$ is obtained by substituting $\bar{u}$ into Eq. (12). Then, the substitution of $F$ into Eq. (11) gives the spring constant $k$. 


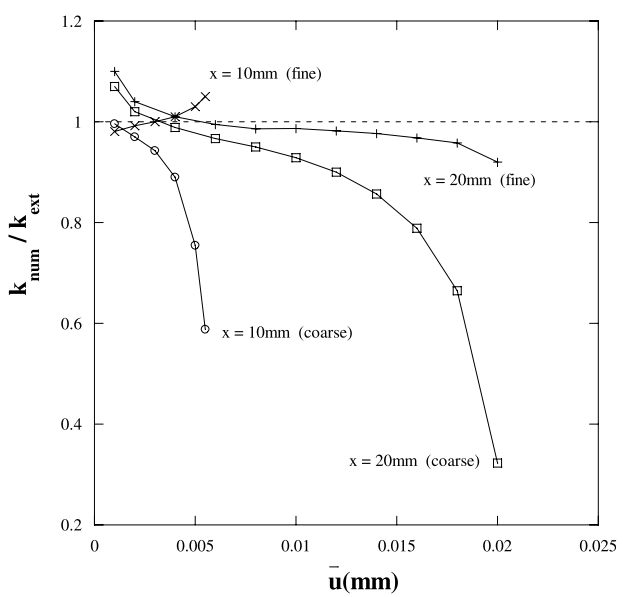

Fig. 7 Comparison between numerical and exact solutions

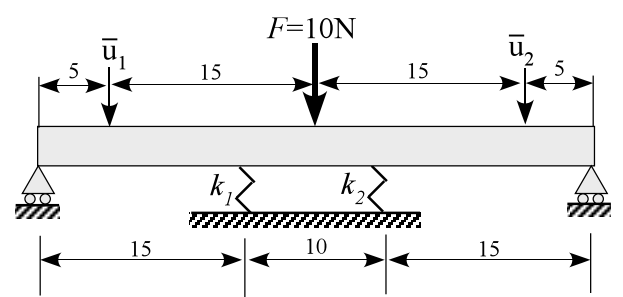

(a) Model-3

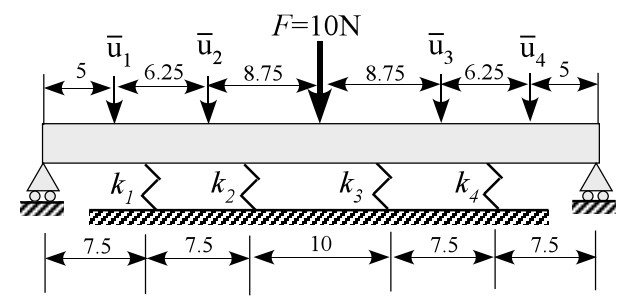

(b) Model-4

Fig. 8 Beams supported at both ends with spring-supported boundaries

\subsection{Comparison with numerical results}

For the two types of models shown in Fig. 4, numerical analyses are conducted on the condition that the beam height $h=5 \mathrm{~mm}, a=20 \mathrm{~mm}, b=10 \mathrm{~mm}$, concentrated load $P=10 \mathrm{~N}$, and Young's modulus $E$ and Poisson's ratio $v$ are 200GPa and 0.3. For both Model-1 and Model-2, plane stress state is assumed and the cantilever is modeled using six quadrilateral elements with identical configuration, which are placed along the length in series. In order to attain a better accuracy with fewer elements, Selected Reduced Integration Scheme ${ }^{(4),(5)}$ (SRI) is used when integrating element matrices, considering that the cantilever deformation is primarily caused by bending. In this case, full integration and reduced integration schemes are applied to the components related to normal stress and shear stress, respectively.

In Figs. 5(a), (b), numerical results of the spring constant $k$ for Model-1 and Model-2 at the supported point are shown for given displacements $\bar{u}$ with various locations $x$. The abscissa represents the magnitude of $\bar{u}$. As $\bar{u}_{i}$ is increased, the spring constant $k$ decreases asymptotically to the x-axis. Exact solutions calculated by Eqs. (11) through (14) are also shown in the figure. The numerical solutions are in good agreement with the exact ones for both Model-1 and Model-2. Accordingly, it has been substantiated that the spring constants obtained by the proposed method only involve the numerical errors traced to the mesh size and numerical integration scheme, as explained in Chapter 3. In Figs.6 (a), (b), numerical results for Model-1 and Model-2 analyzed by use of finer meshes, in which each element is divided into smaller four elements, i.e., a total of twenty-four elements, are compared to the previous ones. It is evident that the FE model with coarse mesh gives a sufficient accuracy over the range of $\bar{u}$ treated here. Next, it is investigated how the magnitude and the location of the given displacement, $\bar{u}$ and $x$, affect the numerical accuracy. Figure 7 shows the ratio of the numerical result to the exact solution of the spring constants, $k_{\text {num }} / k_{\text {ext }}$. The objective is Model- 1 with fine and coarse meshes, and the location of $\bar{u}$ denoted by $x$ is changed as $10 \mathrm{~mm}$ and $20 \mathrm{~mm}$. It is clearly indicated that the fine mesh models give better accuracy over a wide range of $\bar{u}$. Large errors are observed when $\bar{u}$ is small or large. That is because decreasing or increasing $\bar{u}$ gradually approaches the so-called perfect restraint or non-restraint state.

\subsection{Structures supported by multiple springs}

The foundation stiffness of actual large structures can be expressed by means of a number of spring elements. In this section, studied are the structures with more realistic boundary conditions, comparing to those in the previous section. Figures 8(a), (b) show the objective 

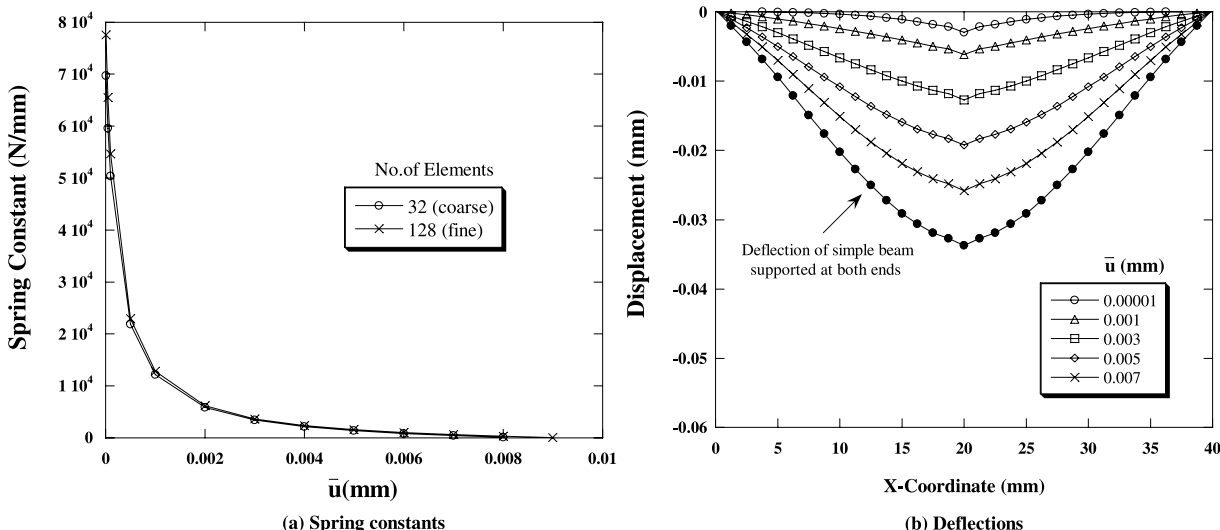

Fig. 9 Numerical results of spring constants and deflections for Model-3

beams, both ends of which are simply supported and its lower surface is sustained by a number of spring elements. Let the beams supported by two springs and four springs be Model-3 and Model-4, respectively. For simplicity, numerical calculations are conducted by assuming that $\bar{u}_{1}=\bar{u}_{2}, k_{1}=k_{2}$ for Model-3 and $\bar{u}_{1}=\bar{u}_{4}, \bar{u}_{2}=\bar{u}_{3}, k_{1}=k_{4}, k_{2}=k_{3}$ for Model-4. The beam height $h$ is $5 \mathrm{~mm}$. The standard numerical model has a total of 32 elements, i.e., 16 elements along the length and 2 elements along the height. Figure 9(a) depicts the numerical results for Model-3. It is shown how the spring constant $k$ at the supported point varies as the given displacement $\bar{u}$ is increased. Numerical results by a finer mesh model with 128 elements are also shown in the figure, as in the case of Fig. 6. The magnitude of $k$ decreases hyperbolically with increasing $\bar{u}$. The number of elements has negligible effect except in the range of small $\bar{u}$ that yields very large $k$. In Fig. 9(b), deflection curves by the fine mesh model are illustrated for varying $\bar{u}$. The bottom line shows the exact solution of a simply supported beam without spring-supported boundaries. Figures 10(a), (b) show the results of Model-4. It is shown how the spring constants $k_{1}$ and $k_{2}$ and the ratio $k_{2} / k_{1}$ vary as $\bar{u}_{2}$ is increased. The figure represents that $k_{1}$ increases almost linearly and $k_{2}$ decrease as $\bar{u}_{2}$ is increased. Although the numerical models employed here are supported by a few number of spring elements, the proposed method can be applied to large and complicated-shaped machines or structures by simply increasing the number of spring elements that represent the foundation stiffness.

\section{Conclusions}

( 1 ) A finite element approach as an inverse problem is proposed to estimate the foundation stiffness of large structures that are modeled as groups of one-dimensional spring elements, by using the same number of measured surface deflections as that of the spring elements.

(2) A cantilever supported by a single spring is chosen as an illustrative example, and the validity of the proposed method has been demonstrated by comparing the numerical results of the spring constant to the corresponding exact solutions.

( 3 ) As an example whose boundary condition is more close to that of actual large structures, a simple beam with its lower surface being supported by multiple spring elements is analyzed, and the relationships between the surface deflection and the foundation stiffness are quantitatively evaluated.

The authors would like to acknowledge Prof. Ishii, Kobe University, for his support on the algebra to solve a set of linear equations with unknown diagonal components.

\section{References}

( 1 ) Kubo, S., Inverse Problems, (1992), pp86-94, Baifukan.

( 2 ) Bui, H.D. (translated by Aoki, S., et. al.), Inverse Problems of Mechanics of Materials, (1994), Shokabo. 
( 3 ) Fukuoka, T., Fundamentals of Numerical Analysis and its Application to Machine Design, Science of Machine, Vol.56, No.4 (2004), pp.619-629.

( 4 ) Kikuchi, N., Finite Element Methods in Mechanics, (1986), pp218-220, Cambridge University Press.

( 5 ) Fukuoka, T. and Senoh, Y., Finite element Simulation of the Strength of the Big End of Diagonally Split Connecting Rod, Transactions of the Japan Society of Mechanical Engineers, Series A, Vol.64, No.617 (1998), pp.104-110. 